\title{
The impact of the quark's monopole properties on the unification of the fundamental physical forces
}

\author{
Engel Roza \\ Philips Research Labs, Eindhoven, The Netherlands (retired) \\ Email: engel.roza@onsbrabantnet.nl
}

\section{Summary}

It is shown that the four fundamental physical forces, i.e. weak interaction, strong interaction, electromagnetism and gravity, all have their origin in the quark as the single true elementary particle. This requires conceiving the quark as a Dirac particle in a pseudo-tachyon mode, which possesses two real dipole moments: the common one associated with its angular momentum and a second one that is polarisable under a scalar potential. This Dirac particle carries a regular charge magnetic monopole without Dirac's string, theorized by Comay. The boson carrier of its field of energy is the gluon showing an exponential decay of its spatial range because of the influence of an omni-present energetic background field, known as the Higgs field, in this article interpreted as the Lambda in Einstein's Field Equation.

Keywords: grand unification; magnetic monopole; pseudo-tachyon; Higgs field

\section{Introduction}

It is probably not by accident that both Cosmology theory and Particle Physics theory rely upon the awareness of an omni-present background energy. In cosmology represented by Einstein's Lambda and in particle physics by the Higgs field. We would have a beautiful physics theory if these two fields of energy are carried by the same energetic constituents. Such a convergence of cosmology and particle physics would mark a major step to unify gravity with quantum mechanics. Because a true unification is still lacking, it might well be that a different mindset from mainstream dogmas is required to obtain this goal. It is my aim to show in this article how the adoption of some uncommon principles, may give a possible route to this. Before detailing these principles and the aimed convergence, it is instructive to mention the most important ones. The major one for cosmology is the view that Einstein's Lambda is not a constant of nature, but instead, a covariant integration constant, independent of space time coordinates, which may depend on attributes of the cosmological system analyzed with the Field Equation [1]. Hence, the Lambda at the level of the universe can be different from the Lambda at the level of a galaxy. In [2], I have given reasons why, starting from a note in Einstein's GR article. Second, the vacuum of the universe is not empty, but contains elementary energetic constituents represented by the Lambda $[1,2]$. The major one for particle physics is the view that a quark is a Dirac particle with two real dipole moments: the conventional one related with its elementary angular momentum $\boldsymbol{h}$ (spin) and a non-conventional one related with its elementary linear momentum $h / c$ (isospin). In a theoretical study, published in Foundations of Physics [3], updated in [4], I 
have shown that Dirac's theory allows a particular modality that shows this property. Furthermore, the quark is conceived as a classical monopole with a potential shielded by a background field from elementary constituents that are regarded as the manifestation of the canonical axiomatic Higgs field. Detailed documentation on the impact of the views that Einstein's Lambda is not a constant of nature and that the quark has two real dipole moments on, respectively, the canonical $\Lambda$ CDM model of cosmology and the canonical Standard Model of particles, can be found in previous work [2,5], which will be just summarized and referenced. This article is aimed to show the merits of these controversial ideas by joining the cosmological view with the particle physics view. At this point it might be useful to read the discussion paragraph (10) first, before studying the main line. The article starts (in paragraph 2) with a summary of a structural model for the basic quark-antiquark junction that composes the archetype meson, such as made possible after modelling quarks as classical monopoles in an ambient energetic background field. Such a structure is the result of an equilibrium of forces between a repulsive force and an attracting force. Whereas in an electromagnetic monopole structure, such as a positronium, the monopole attraction is neutralized by the centrifugal repulsion, the monopole fields of the quarks in hadron structures are balanced by the fields evoked by their real valued second dipole moments. In the Appendix this unexpected property is put into perspective between three Dirac particle modalities. Paragraph 3 deals with the quark's monopole field, thereby showing that its strength can be equivalently expressed in terms of energy or, in analogy with electromagnetism, in terms of an unknown nuclear charge. Paragraph 4 addresses the question if this nuclear charge can be identified as a magnetic monopole charge. In paragraphs 6 and 7 the relationship is discussed between the energetic ambient field of particle physics (the Higgs field) and the energetic ambient field in cosmology (the fluid embodied by Einstein's Lambda), thereby showing the possible identity of their constituents. The final part is a discussion, a corollary and a conclusion.

\section{Summary of earlier work on particle physics}

The work to be described in this document is an extension on earlier work, in which hadrons have been described in terms of quarks that are conceived as Dirac particles of a particular type that possess the unique property of having a polarisable dipole moment in scalar potential field [5]. It has been shown and justified that the quark can be modelled as an energetic pointlike particle that erupts an energetic field $\Phi(r)$, which can generically be expressed as,

$$
\Phi_{F}(r)=\frac{\Phi_{F 0}}{\lambda r}
$$

in which $\Phi_{F 0}$ is a strength parameter in units of energy and in which $\lambda$ is a normalization parameter with dimension $\left[\mathrm{m}^{-1}\right]$. The polarisable dipole moment is responsible for an 
additional near field that along the direction of the dipole axis has the format $\Phi_{N}(x)$, which can be generically be expressed as,

$\Phi_{N}(x)=\frac{\Phi_{N 0}}{(\lambda x)^{2}}$

The two fields can be combined into a single field expression. To explain the short spatial range of nuclear forces, it has been assumed that the fields are shielded by an energetic background field in a similar way as the Coulomb field of a charged particle in an ionic plasma is shielded by the Debije effect. As a result from these two contributions, the potential field of a quark in this background field can be generically expressed along the axis of the dipole moment as,

$\Phi(x)=\Phi_{0} \exp (-\lambda x)\left\{\frac{1}{(\lambda x)^{2}}-w \frac{1}{\lambda x}\right\}$

in which $w$ is a dimensionless weighing factor that relates the far field with the near field. The identification of the background field with the Higgs field as defined in the Standard Model has enabled the assessment of a numerical value $w \approx 1 / 0.55$. The shape of the potential function, shown by figure $1 \mathrm{a}$, is known as "the liquid drop model" [3]. This model, conceived in 1928 by Ganov [7] for the internucleon potential, has been adopted, apart from me [8], by Comay for the interaction between the two quarks in a pion [9]. As proven in $[8,10]$, It can be retrieved from the Higgs Lagrangian [11],

$$
U(\Phi)=-\frac{\mu_{H}^{2}}{2} \Phi^{2}+\frac{\lambda_{H}^{2}}{4} \Phi^{4}
$$

shown in figure $1 \mathrm{~b}$, nicknamed as the "Mexican hat model", owing to its shape if rotated around the vertical axis. This quark model enables to conceive the archetype meson (pion) as a structure shown in figure 2 . In this structure, the quark is coupled by a dimensionless coupling factor to the field of the antiquark. In the center-of-mass frame, the relativistic twobody structure can be modelled as a non-relativistic one-body $a(n)$ harmonic oscillator that can be described by a Schrödinger type wave function $\psi$ of the center-of-mass in the wave function equation,

$-\frac{\hbar^{2}}{2 m_{m}} \frac{\mathrm{d}^{2} \psi}{\mathrm{dx}^{2}}+g \Phi_{0}\left\{k_{0}+k_{2} \lambda^{2} x^{2}+\ldots.\right\} \psi=E \psi$

in which $g$ is a dimensionless coupling factor. This represents an anharmonic quantum mechanical oscillator characterized by quantum steps $\hbar \omega$ related with the effective mass $m_{m}$, such that

$\frac{1}{2} m_{m} \omega^{2}=g \Phi_{0} k_{2} \lambda^{2} \rightarrow \frac{m_{m}^{\prime}(\hbar \omega)^{2}}{(\hbar c)^{2}}=2 g \Phi_{0} k_{2} \lambda^{2}$ 

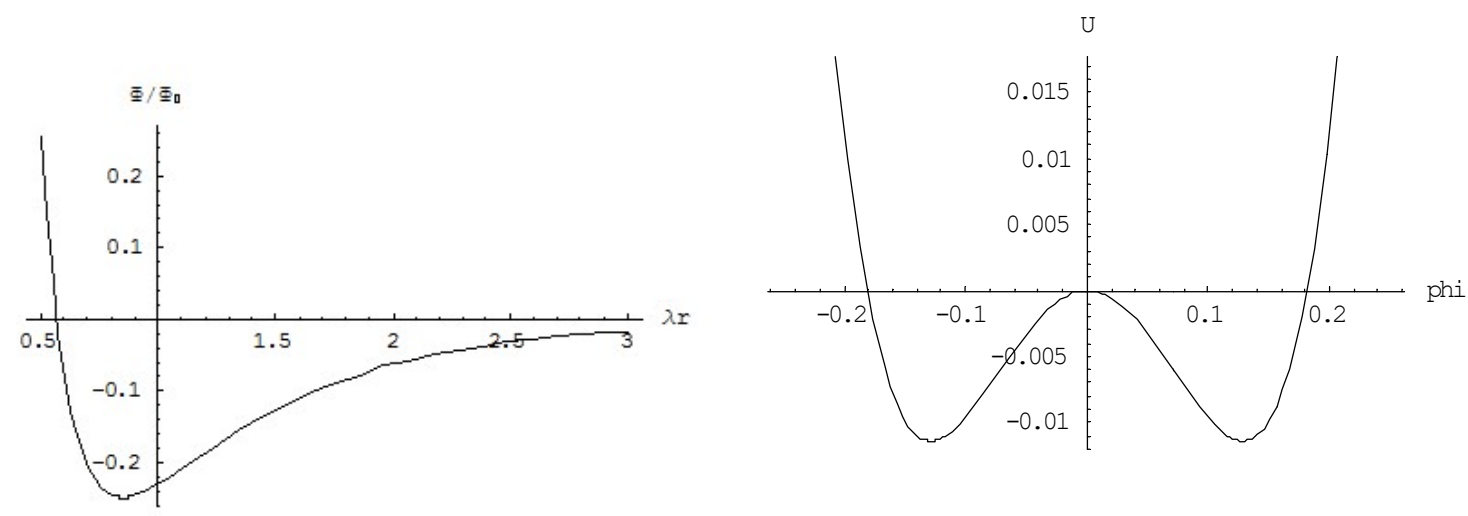

Fig. 1. (Left) The quark's scalar field $\Phi / \Phi_{0}$ as a function of the normalized radius $\lambda x$; (Right) The field's Lagrangian $U_{H}(\Phi)=-U(\Phi)$ retrieved from the spatial expression. .

Conventionally, $m_{m}^{\prime}=m_{m} c^{2}$ is the energy of the central mass of the oscillator. In this case, the mass does not represent the individual masses of the two bodies, but it is an equivalent mass that captures the energy of the field. As usual, $\omega$ is related with the vibration energy $E_{n}=(n+1 / 2) \hbar \omega$. The dimensionless constant $k_{0}$ is a measure for the binding energy between the two bodies. The dimensionless constant $k_{2}$ is determined by the curvature of the potential in the center of mass. These values can be straightforwardly calculated from (3) as $k_{0}=-1 / 2$ and $k_{2}=2.36$ [5].

Considering that the pion decays into a fermion via the weak interaction boson, the boson $\hbar \omega$ can be equated with the weak interaction boson. Hence,

$\hbar \omega=\hbar \omega_{W}$.

Its value $\hbar \omega_{W}=80.4 \mathrm{GeV}$ represents the relativistic value of the non-relativistic lab frame rest mass of the pion $\left(m_{\pi}^{\prime}=m_{\pi} c^{2} \approx 140 \mathrm{MeV}\right)$.

The oscillator settles itself into minimum energy condition. This is established under a particular spacing $2 d$ between the two quarks, such that $d_{\min }^{\prime}=d \lambda=0.853$. In this condition two important relationships can be derived. These are, respectively,

$\hbar \omega_{W}=2\left|k_{0}\right| g \Phi_{0}=g \Phi_{0}$

$\frac{g \Phi_{0}}{\lambda}=\frac{\alpha \pi(\hbar c)}{2 d_{\min }^{\prime}}$

in which $\alpha$ is a dimensionless constant of order 1 , the value of which has been calculated as $\alpha \approx 0.69$ [10].

The simple anharmonic oscillator model described by (5) enables the mass spectrum calculation of the pseudoscalar mesons as excitations from the pion state. The excitation 
mechanism stops beyond the bottom quark due to the loss of binding energy. The mass spectrum calculation of the vector mesons requires the inclusion of the impact of the nuclear spin shown in the upper part of figure 2. A spin flip marks the difference between the pseudoscalar pion and the vector type sisters rho. The massive energy difference $\Delta E$ between the two types is a consequence of a spin-spin interaction process. It is of a similar nature as the analysis of the interaction process between the electron and the proton nucleus in a hydrogen atom. This requires a detailed quantum mechanical computation. Recognizing, though, that this is essentially a bosonic process, allows, in retrospect, a surprising simple approach. The step to be taken is conceiving the massive energy difference $\Delta E$ as a result of a bosonic interaction mediated by $Z$ bosons in virtual state. Because of the asymmetry in the spin-spin interaction $\left(-3 \hbar^{2} / 4\right.$ and $\left.+\hbar^{2} / 4\right)$, we have,

$m_{\pi}^{\prime}=2 m_{u}^{\prime}-3 m_{Z}^{\prime \prime}$ and $m_{\rho}^{\prime}=2 m_{u}^{\prime}+m_{Z}^{\prime \prime}$,

in which $m_{\pi}^{\prime}, m_{\rho}^{\prime}, m_{u}^{\prime}$ and $m_{Z}^{\prime \prime}$ are the energies of, respectively, the rest masses of pion and the rho meson, the constituent massive energy of the $u / d$ quark and the energy of the $Z$ boson in virtual state in the rest frame of mesons. The statement that the energy of the rest mass of the pion is equal to the non-relativistic equivalent of the energy of the $W$ boson enables to calculate the energy $m_{Z}^{\prime \prime}$ of the $Z$ boson in virtual state as,

$m_{Z}^{\prime \prime}=m_{Z}^{\prime}\left(\frac{m_{\pi}^{\prime}}{m_{W}^{\prime}}\right)$.

Under use of (10) and (11), the constituent rest mass energy $m_{u}^{\prime}$ of the $u / d$ quark is calculated as

$m_{u}^{\prime}=\frac{1}{2} m_{\pi}^{\prime}\left(1+3 \frac{m_{Z}^{\prime}}{m_{W}^{\prime}}\right)$

From (11), the energy of the constituent massive energy of the $u / d$ quark is, under consideration of the energetic values of the weak interaction bosons $m_{W}^{\prime}=80.4 \mathrm{GeV}$ and $m_{Z}^{\prime}=91.2 \mathrm{GeV}$, under adoption of the rest mass of the pion $m_{\pi} \approx 140 \mathrm{MeV} / \mathrm{c}^{2}$, calculated as $308 \mathrm{MeV}$. Under use of this value, the energy of the rho meson is calculated from (10) and (11) as $m_{\rho}^{\prime}=775 \mathrm{MeV}$. This is a perfect fit with experimental evidence. It is probably useful to note that, in spite of its role as boson in virtual state, the $Z$ boson does not necessarily show up as free particle in the strong decay process of vector-type mesons into pseudoscalar ones. All together, this view enables to calculate the mass spectrum of the mesons with high precision [5]. 


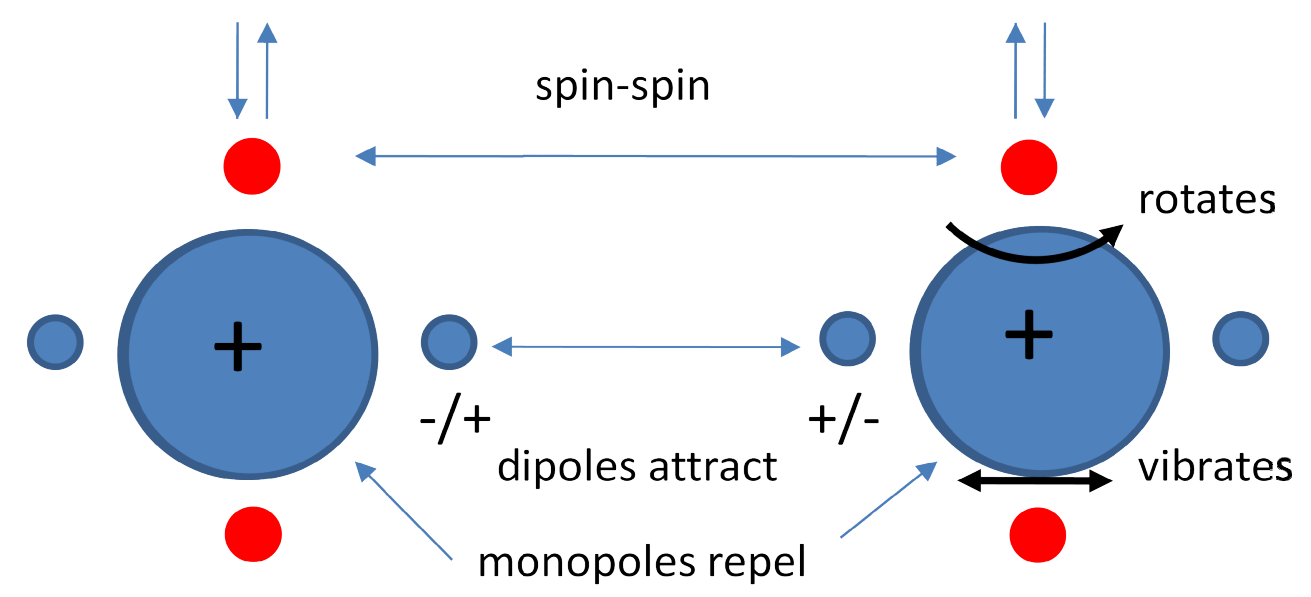

Fig. 2. A quark has two real dipole moments, hence two dipoles. One of these (horizontally visualized) is polarisable in a scalar potential field. The other one (vertically visualized) is not. The dipole moments are subject to spin statistics. However, the polarity of the horizontal one is restrained by the bond: the horizontal dipoles are only oriented in the same direction: either inward to the centre or outward from the centre.

In this model, the role of the Higgs field is represented by the shield parameter $\lambda$. A comparison of this structural model with the Standard Model [5], has revealed the relationship,

$m_{H}^{\prime}=2 \lambda(\hbar c)$.

In fact, the Higgs shows up as the signature of two gluons rather than as an individual particle. More particularly, the quark-gluon relationship is seen as the nuclear equivalent of the electron-photon relationship. It means that the gluon should be interpreted as the boson associated with the quark's far field (1). Such boson is subject to the Proca-type wave equation,

$\frac{1}{c^{2}} \frac{\partial^{2} r \Phi}{\partial t^{2}}-\frac{\partial^{2}}{\partial r^{2}} r \Phi+\lambda^{2} r \Phi=\rho_{H}(r, t)$

in which $\rho_{H}(r, t)$ is a Dirac-type pointlike source that can be expressed as,

$\rho_{H}(r, t)=4 \pi r \frac{\Phi_{0}}{\lambda} \delta^{3}(r) H(t)$

in which $H(t)$ and $\delta(r)$, respectively, are Heaviside's step function and Dirac's delta function.

Figure 3 shows the solution of the gluon's wave function in a graphical format. Unlike a gamma photon, the gluon is subject to dispersion. The dispersion is due to the $\lambda^{2}$ term in the Proca wave equation (12). This term is a consequence of the energetic ambient field, known as the Higgs field. 


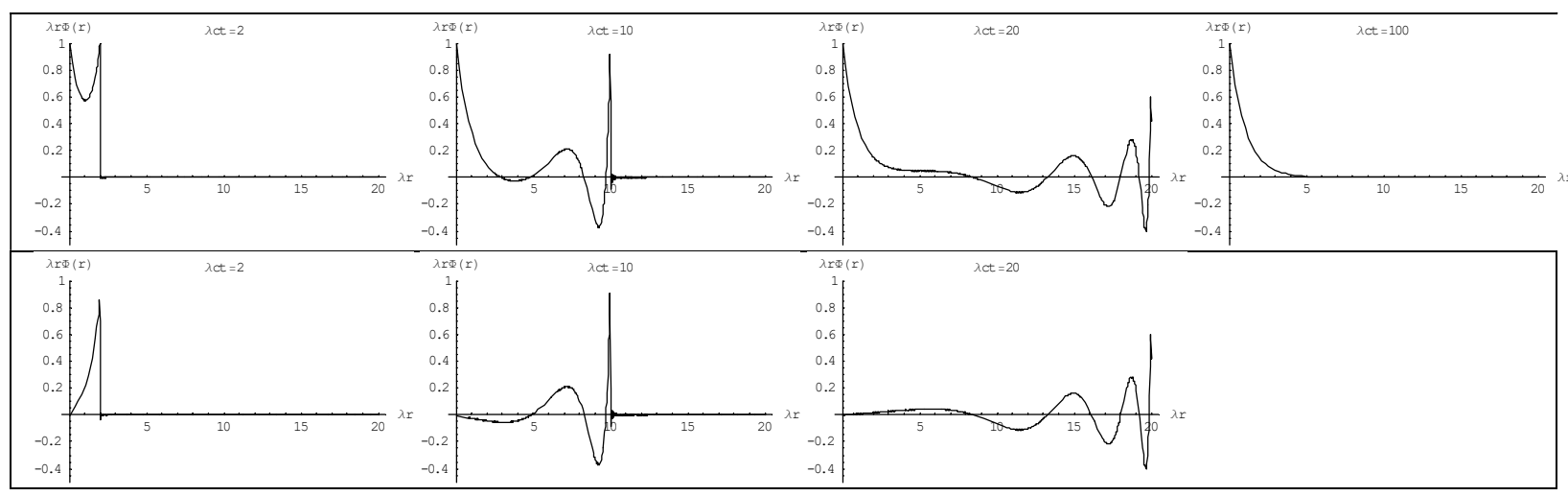

Fig.3. The building of the quark's potential far field as a result of a sudden energy eruption from its source. The field is the sum of the steady solution shown at the right and the transient pulse shown in the lower part of the figure. This pulse is the actual gluon. It propagates at light speed and it eventually disappears as a result of dispersion. If $\lambda$ is zero, the transient is a never disappearing gamma photon and the stationary situation is shown by an unfinished rectangular shape of the upper most right graph. Note that the field is represented by $r \Phi(r)$.

\section{The monopole properties of the quark}

So far in this description, a generic energetic potential $\Phi$ has been assigned to the quark, to which an identical other one couples with a dimensionless coupling factor $g$, such that the interaction force $F$ is expressed as,

$$
F=g \frac{\partial}{\partial r} \frac{\Phi_{0}}{r^{\prime}} ; \quad r^{\prime}=r \lambda
$$

in which $\lambda$ is a normalization quantity making $r^{\prime}$ dimensionless. Because of the degree of freedom in the invariant product $g \Phi_{0}$, the coupling factor $g$ has been set to the square root of the electromagnetic fine structure constant $\alpha_{e}$, such that $g=\sqrt{\alpha_{e}}=1 / \sqrt{137}$.

Doing so similarly for the interaction between two electrons, we would have,

$$
F=g \frac{\partial}{\partial r} \frac{\Phi_{0}^{\prime}}{r^{\prime}}=\frac{e^{2}}{4 \pi \varepsilon_{0}} \frac{\partial}{\partial r} \frac{1}{r}=\frac{e^{2}}{4 \pi \varepsilon_{0}} \frac{\partial}{\partial r} \frac{\lambda}{r^{\prime}}=\alpha_{e} \hbar c \lambda \rightarrow \Phi_{0}^{\prime}=g(\hbar c) \lambda .
$$

Doing so for the interaction between two unknown nuclear charges $u$, we would have,

$$
F=g \frac{\partial}{\partial r} \frac{\Phi_{0}}{r^{\prime}}=u^{2} G_{q u} \frac{\partial}{\partial r} \frac{1}{r}=u^{2} G_{q u} \frac{\partial}{\partial r} \frac{\lambda}{r^{\prime}} \rightarrow \Phi_{0}=N_{q u} g(\hbar c) \lambda ; \quad N_{q u}=\frac{u^{2} G_{q u}}{g(\hbar c)}
$$

In this picture, the quantity $N_{q u}$ is the factor that expresses the excess strength over the electrical strength. It can be calculated by comparing the far field force $F_{F}$ evoked by a quark with the electromagnetic force $F_{e}$ evoked by an electron $e$. Generally, 


$$
F_{e}=-e \frac{\partial}{\partial r} \frac{e}{4 \pi \varepsilon_{0} r} \quad \text { and } \quad F_{F}=-g \frac{\partial}{\partial r} w \Phi_{0} \frac{\exp (-\lambda r)}{\lambda r}
$$

There is no reason why these forces would be the same. What is clear, however, is, that $g w \Phi_{0} / \lambda$ plays a similar role as $e^{2} /\left(4 \pi \varepsilon_{0}\right)$, i.e.,

$$
\frac{e^{2}}{4 \pi \varepsilon_{0}} \leftrightarrow w \frac{g \Phi_{0}}{\lambda}
$$

It means that the electric force from certain electric charge $q_{e}$ is equivalent with a nuclear force such that

$$
\frac{q_{e}^{2}}{4 \pi \varepsilon_{0}}=w \frac{g \Phi_{0}}{\lambda} .
$$

Hence, from (9), (12) and (20),

$$
q_{e}^{2}=4 \pi \varepsilon_{0} w \frac{m_{W}^{\prime}}{m_{H}^{\prime}}(2 \hbar c)=\frac{2 w}{g^{2}}\left(\frac{m_{W}^{\prime}}{m_{H}^{\prime}}\right) e^{2}
$$

A numerical evaluation of this expression $\left(m_{W}^{\prime}=80.4 \mathrm{GeV} ; m_{H}^{\prime}=125 \mathrm{GeV}, g^{2}=1 / 137, w=\right.$ $1 / 0.55$ ) reveals that the far field nuclear force between two archetype quarks is about equivalent with the electric force between 18 electrons.

The parallel between the nuclear charge $u$ and the electric charge $q_{e}$ as shown by (16-17) evokes the suggestion that the nuclear energy might have an electromagnetic origin. A bold hypothesis is supposing that the quark might be a magnetic monopole $q_{m}$ with an equivalent strength of 18 electrons. Considering the quark as a Dirac particle with magnetic monopole properties instead of electric monopole properties (like an electron), it must have a real electrical dipole moment, similarly as the electron has a real magnetic one.

\section{Comay's monopole versus Dirac's monopole}

Hence, let us proceed with the hypothesis that the quark is a magnetic monopole. As is well known, the magnetic monopole concept is based upon a generalization of Maxwell's equations. Let us consider the Gaussian part,

$$
\nabla \cdot \mathbf{E}=\frac{\rho_{e}}{\varepsilon_{0}} ; \quad \nabla \cdot \mathbf{B}=\mu_{0} \rho_{m}
$$

in which $\rho_{e}$ is electrical space charge and $\rho_{m}$ is hypothetical magnetic space charge. Solving these equations under pointlike conditions $q_{e}$ and $q_{m}$ for the space charges, 
$\rho_{e}=q_{e} \delta^{3}(r) ; \rho_{m}=q_{\mu} \delta^{3}(r)$

the resulting field strength expressions have the magnitude formats,

$E=\frac{1}{4 \pi \varepsilon_{0}} \frac{q_{e}}{r^{2}} ; \quad B=\frac{\mu_{0}}{4 \pi} \frac{q_{m}}{r^{2}}$.

Whereas an "electron-type" Dirac particle with a pointlike electric charge $q_{e}$ has a real magnetic dipole moment $\boldsymbol{\mu}_{\mathrm{m}}$ and an imaginary electric dipole moment, an "electron-type" Dirac particle with a pointlike magnetic charge $q_{m}$ has a real electric dipole moment $\boldsymbol{\mu}_{\mathrm{e}}$ and an imaginary magnetic dipole moment. A (non-electron) quark-type Dirac particle, with its two real dipole moments and a nuclear charge interpreted as magnetic charge, has a real electric moment as well as a real magnetic moment. Inherited from electromagnetism, the eigen values are, respectively,

$\mu_{e}=\frac{q_{m}}{2 m_{0}} \hbar ; \mu_{m}=\frac{q_{m}}{2 m_{0}} \frac{\hbar}{c}$.

From (21) and (22), the magnetic charge is calculated as,

$q_{m}^{2}=\frac{2 w}{g^{2}}\left(\frac{m_{W}^{\prime}}{m_{H}^{\prime}}\right) e^{2} c^{2}$

This allows the assessment of its numerical value as $q_{m}=8.64 \times 10^{-10} \mathrm{~A} \mathrm{~m}$. Note that this value is frame invariant. The value is well below the minimum value of the magnetic monopole as derived in Dirac's classic paper [15]. Dirac's monopole is constrained by the condition,

$q_{m} e=n\left(\frac{2 \pi \hbar}{\mu_{0}}\right)$

in which $n$ is a natural number. This gives a minimum value for the magnetic monopole as $3.29 \times 10^{-9} \mathrm{~A} \mathrm{~m}$, which is significantly larger than the calculated value for the quark. This seems to exclude the possibility that the quark is a magnetic monopole. However, it has to be taken into account that Dirac's monopole is driven by the wish to prove the quantized nature of electric charge. As pointed out by Comay $[9,16,17,18]$ in his Regular Charge Monopole Theory (RCMT), this wish has spoiled the symmetry of Dirac's monopole theory. Dirac's theory as well as Comay's theory is fully symmetrical under the substitutions $\mathbf{E} \rightarrow \mathbf{B} ; \mathbf{B} \rightarrow-\mathbf{E}$ and $q_{e} \rightarrow q_{m} ; q_{m} \rightarrow-q_{e}$. However, whereas full symmetry would require a vector potential $\mathbf{A}$ such that $\mathbf{E}=\nabla \times \mathbf{A}$, Dirac maintained $\mathbf{B}=\nabla \times \mathbf{A}$, under allowance of a string singularity. This difference makes Dirac's monopole theory asymmetrical, while Comay's monopole theory is fully symmetrical. As long as magnetic monopoles and electric monopoles (i.e. electric pointlike charges) are mutually exclusive, Comay's theory is the true 
equivalent of the canonical Maxwell theory. Its strength comes forward in conditions of simultaneous presence of magnetic monopoles and electric monopoles. While Dirac's monopole theory is based upon a hypothetical interaction between the two, Comay has proven that such interaction is an inconsistency by theory [17,19]. Comay's ("magnetoelectric") monopole can be described by Maxwell's equations, but its fields are disjunct from the ones evoked by an electric ("electromagnetic") monopole.

Adopting this view in more explicit terms means the adoption of the existence of magnetoelectrism next to electro-magnetism, in which the quark is supposed as being a magnetic monopole in a magneto-electric field. This magneto-electric field is governed by a vector potential, in which $\mathbf{E}=\nabla \times \mathbf{A}$. Later in this text it will be shown that this magneto-electric field may evoke an electro-magnetic field. For the moment we conclude that the quark, apart from being a special-type Dirac particle with two real dipole moments, is a magnetic monopole in a magneto-electric field. We may proceed now by assigning to the quark a magnetic monopole field $\mathbf{B}(r)$ associated with, respectively, a magnetic dipole field $\mathbf{B}_{d}(r)$ and a electric dipole field $\mathbf{E}_{d}(r)$. Both dipole fields are real. Hence,

$$
\begin{aligned}
& \mathbf{B}(r)=\frac{\mu_{0}}{4 \pi} q_{m} \frac{\hat{\mathbf{r}}}{r^{2}}, \\
& \mathbf{B}_{d}(r)=\frac{\mu_{0}}{4 \pi}\left\{\frac{1}{r^{3}}\left[3\left(\boldsymbol{\mu}_{m} \cdot \hat{\mathbf{r}}\right)-\boldsymbol{\mu}_{m}\right]+\frac{8 \pi}{3} \boldsymbol{\mu}_{m} \delta^{3}(r)\right\}, \\
& \mathbf{E}_{d}(r)=\frac{1}{4 \pi \varepsilon_{0}}\left\{\frac{1}{r^{3}}\left[3\left(\boldsymbol{\mu}_{\mathrm{e}} \cdot \hat{\mathbf{r}}\right)-\boldsymbol{\mu}_{\mathrm{e}}\right]+\frac{8 \pi}{3} \boldsymbol{\mu}_{\mathrm{e}} \delta^{3}(r)\right\},
\end{aligned}
$$

in which $\hat{\mathbf{r}}$ is the unit vector in $r$ direction.

One may expect that the magnetic dipoles will align along the direction of the field between the two monopoles. If the quarks are magnetic monopoles, the quarks will attract, because if the quark has a positive magnetic charge, the antiquark has a negative charge. Hence, figure 2 requires a somewhat different interpretation, like shown in figure 4 . This interpretation has no effect on the theory developed in [5].

The magnetic potential $\Phi_{m}(x)$ along the dipole axis can be expanded as,

$$
\Phi_{m}(x)=-\frac{\mu_{0}}{4 \pi} \frac{q_{m}}{x}+\frac{\mu_{0}}{4 \pi}\left(\frac{q_{m}}{2 m_{0}} \frac{\hbar}{c}\right)\left(\frac{1}{x^{2}}+\ldots . .\right)
$$

The first right-hand term is the magnetic monopole potential and the second right-hand term is the magnetic dipole potential. The potential can be rewritten by approximation as,

$$
\Phi_{m}(x)=\Phi_{0}^{m}\left\{\frac{1}{(\lambda x)^{2}}-w \frac{1}{\lambda x}\right\}, \text { with }
$$


$\Phi_{0}^{m}=\frac{\mu_{0}}{4 \pi}\left(\frac{q_{m}}{2 m_{0}} \frac{\hbar}{c}\right) \lambda^{2}$ and $w=\frac{2 m_{0} c^{2}}{\hbar c \lambda}$.

Equating

$g \Phi_{0}=w q_{m} \Phi_{0}^{m}$

allows to obtain an expression for the generic potential $\Phi_{0}$, such that the potential field of the quark can be read as (3), in which, under consideration of (25) and (30),

$\Phi_{0}=\frac{1}{g} \frac{\mu_{0}}{4 \pi} q_{m}^{2} \lambda ; q_{m}^{2}=\frac{2 w}{g^{2}}\left(\frac{m_{W}^{\prime}}{m_{H}^{\prime}}\right) e^{2} c^{2} ; w=\frac{2 m_{0} c^{2}}{\hbar c \lambda}$

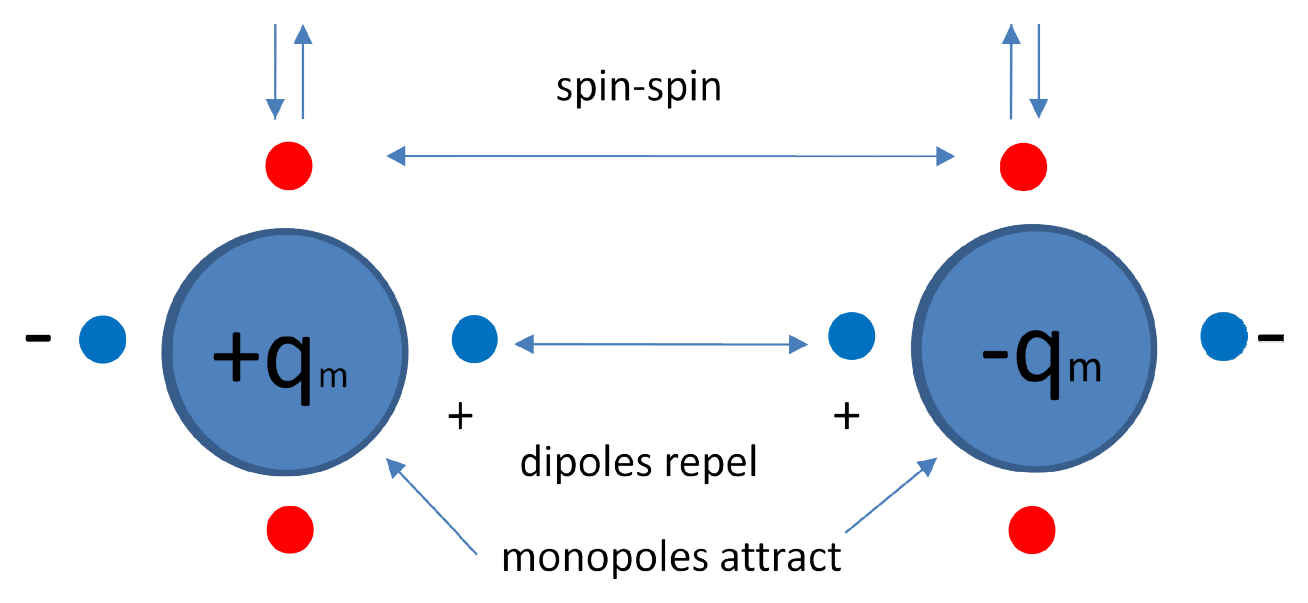

Fig. 4. Electromagnetic interpretation of figure 1.

\section{Electric charge}

It has been shown in $[5,10]$ that the bond between two and three Dirac particles with polarisable dipole moments in a scalar field is the cradle of baryonic energy, because the center-of-energy of the two-particle and three-particle bonds shows the characteristics of the energy-stress tensor in Einstein's Field Equation for gravity. It is an empirical fact that these bonds are electrically charged. We have also concluded that the nuclear energy from a quark can be interpreted in terms of a nuclear charge and that this nuclear charge can be hypothesized as magnetic monopole in an magneto-electric field (Comay's RCMT). Adopting the view that the quark is a special-type Dirac particle with two real dipole moments, one of the two is a magnetic dipole and the other one is an electric dipole. It might well be that the magnetic dipole coincides with the magnetic dipole of an electric charge in a electromagnetic field. If so, the bond between the quark and the antiquark not only generates a center-of baryonic energy, but it also generates the electric charge that takes part of this baryonic energy. Figure 5 shows the concept. 
To investigate the hypothesis, let us equate the magnetic dipole momentum $\mu_{m}^{m}$ of a monopole $q_{m}$ with unknown mass $m_{0}$ with the magnetic dipole $\mu_{m}^{e}$ of an electric charge $q_{e}$ with unknown mass $m_{e}$. Hence,

$\frac{q_{m}}{2 m_{0}} \frac{\hbar}{c}=\frac{q_{e}}{2 m_{e}} \hbar \rightarrow q_{m}=\frac{m_{0}}{m_{e}} q_{e} c$

Invoking (32) and applying it to (33) gives a relationship between $m_{0}$ and $m_{e}$

$$
\begin{aligned}
& q_{m}=\frac{m_{0}}{m_{e}} q_{e} c=\left\{\frac{2 w}{g^{2}}\left(\frac{m_{W}^{\prime}}{m_{H}^{\prime}}\right) e^{2} c^{2}\right\}^{1 / 2} \rightarrow \\
& \frac{m_{0}}{m_{e}}=\frac{e}{q_{e}}\left\{\frac{2 w}{g^{2}} \frac{m_{W}^{\prime}}{m_{H}^{\prime}}\right\}^{1 / 2} .
\end{aligned}
$$

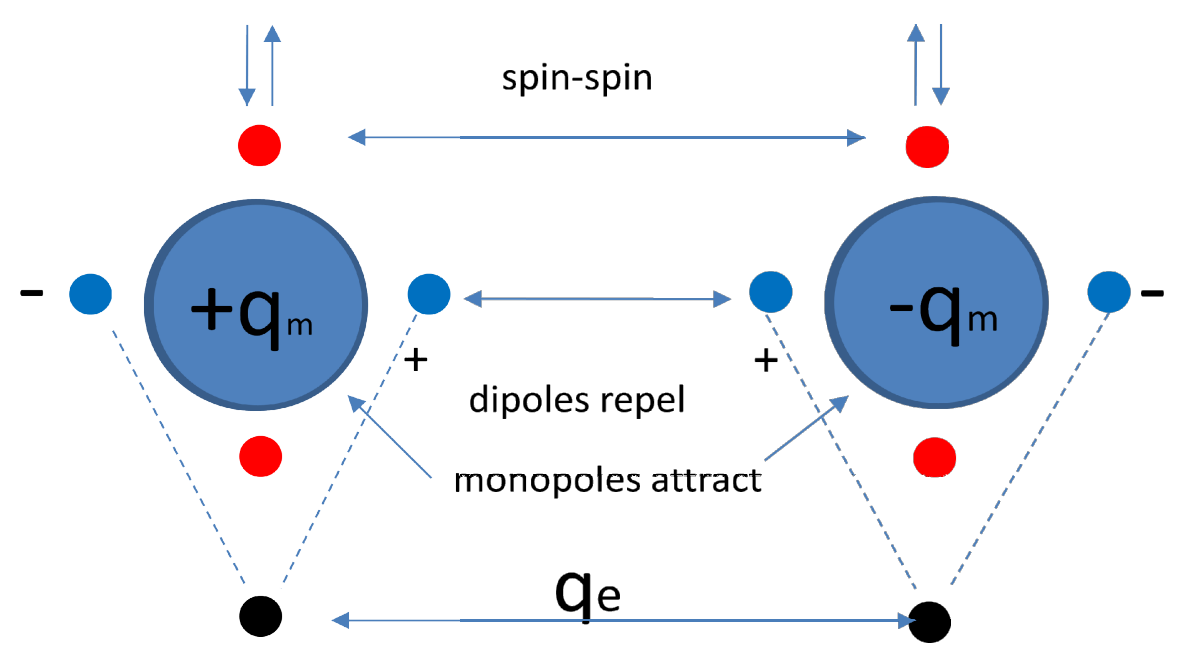

Fig.5: Hypothetical equivalence of the quark's polarisable linear dipole moment with the magnetic dipole moment of its electric charge attribute.

Because the electric charge of the pion equals the elementary charge $e$ and because this charge is composed by two contributions $q_{e}$, one might suppose that for symmetry reasons $q_{e}=e / 2$. In a minimum state of energy, the spatial distance between the quarks is at maximum. Under this condition the spatial distribution of charge would evoke a repulsive effect under the creation of two kernels of equal charge, thereby making a charged pion, whereas a slightly higher state of energy would occur under a distribution into two kernels with opposite charge, thereby making a neutral pion.

The semantics of the masses $m_{0}$ and $m_{e}$ are different. The mass $m_{0}$ is the constituent mass in the (an)harmonic oscillator model of the pion. Let $m_{\pi}^{\prime}$ be the energy of the pion's rest mass, determined from its decay products. This decay is mediated by the weak interaction boson $\hbar \omega_{W}$. Hence, It is fair to suppose that its (relativistic) energy represents the equivalent of the non-relativistic lab frame rest mass, i.e., $\hbar \omega_{W} \rightarrow m_{\pi}^{\prime}$. The energy of the weak interaction 
boson is composed by two components. These are the binding energy between the quarks and the equivalent constituent energies from the two quarks. Considering that the ground state energy of the (an)harmonic oscillator that they build amounts to $\hbar \omega_{W} / 2$, the other half has to be attributed to the constituting masses. Because the two masses constitute a one-body equivalent of a two-body oscillator, and considering that the effective mass $m$ of the single body is made up as, $m=m_{1} m_{2} /\left(m_{1}+m_{2}\right)$, the constituent rest mass energies $m_{0}^{\prime}$ of the quarks in the pion equivalent of the positron can be established from,

$$
\frac{\hbar \omega_{W}}{2}=\frac{m_{\pi}^{\prime}}{2}=\frac{m_{0}^{\prime 2}}{2 m_{0}^{\prime}} \rightarrow m_{0}^{\prime}=m_{\pi}^{\prime} .
$$

The mass $m_{e}$ is different. It is the "bare mass" of the quark. Hence, from (34) and (35),

$$
m_{e}^{\prime}=g \frac{m_{\pi}^{\prime}}{2} \sqrt{\frac{1}{2 w} \frac{m_{H}^{\prime}}{m_{W}^{\prime}}} .
$$

From $m_{W}^{\prime}=80.4 \mathrm{GeV} ; m_{H}^{\prime}=125 \mathrm{GeV}, g^{2}=1 / 137, w=1 / 0.55$ and $m_{\pi}^{\prime}=140 \mathrm{MeV}$, the energy of this bare mass is calculated from (34) and (35) as $m_{e}^{\prime} \approx 3.9 \mathrm{MeV}$. It should not be confused with the $4.6 \mathrm{MeV}$ massive energy difference between a charged pion and a neutral pion. The latter is a consequence of the spin-spin interaction between the electrical dipole moments of the bare masses [41].

Unfortunately, no experimental evidence is available for the quark's bare mass. The result shown is about the same as the "current" masses that are used as input values for lattice QCD as estimated by the Particle Data Group (PDG), [36]. In the context of lattice QCD, the current masses of the quarks in a pion are distinguished from their "dressed" masses. The energy comprised in the dressed masses is made up by the nuclear interaction forces between the quarks. What is left over is said to be comprised in the current mass. Curiously, the lattice QCD model does not allow a differentiation between the bare masses to the $u$ quark and the $d$ quark. Instead a average value $\bar{m}_{u d}=\left(m_{u}+m_{d}\right) / 2$ is obtained with a value $\bar{m}_{u d}=(3.410 \pm 0.043) \mathrm{MeV} / \mathrm{c}^{2}$. This value compares rather well with the $3.9 \mathrm{MeV} / \mathrm{c}^{2}$, as found from (34)-(35).

(Different from a split of the elementary charge into two halves, the Standard Model of particle physics favors a split of $1 / 3$ and $2 / 3$. Such an asymmetrical split is justified from problems in dividing the elementary charge over three quarks, such as required for baryons. Like shown in [5], but left beyond the scope of this text, the electric charge issue is related with isospin, which in the context of the Standard Model is accepted as an empirical phenomenon, while it is actually a result of the unrecognized polarisable dipole moment of the quark under a scalar potential. This structural interpretation allows the equal distribution of the elementary charge among the quarks. It requires the recognition of an orbital spin in baryon structures. Joining this spin to the isospins of the quarks not only allows to adopt a uniform charge distribution among the quarks, but it also explains the 
quantized nature of electric charge because of the quantized nature of spin. There is neither a need for Dirac's monopole to explain it, nor for generalized Dirac monopoles $[20,21,22]$.)

It is fair to conclude that the magnetic monopoles in the pion have ("Gilbertian") magnetic dipole moments that create magnetic fields in a force balanced structure with the magnetic monopole fields, while being at the same time the "Amperian" dipole moment of kernels of electric charge. The classification "Gilbertian" (for magneto-electrism) and "Amperian" (for electro-magnetism) is from Mc. Donald [17]. Rather than identifying the quark as an electroweak monopole [23], it may be seen as a revival of Schwingers's suggestion from 1969 that a quark is a dyon, i.e., both an electric monopole and a magnetic monopole [24]. However, whereas Schwinger proposed his dyon to explain the (quasi) stable hadron structures from a balance between an attracting magnetic force and a repelling electric force (or vice versa), the balance in the structure shown in figure 5 is obtained by the balance between the monopole field and the field evoked by the polarizable dipole moment under a scalar potential. Electric charge pops up as a minor side effect, hence not as a gluing force. This marks a fundamental difference between Schwinger's dyon (abandoned and replaced by $Q C D$ ) and the structure shown in figure 5 .

\section{The gravitational background field (Einstein's Lambda)}

An essential element in the theory surveyed so far is the presupposed existence of an energetic background field. Here, we meet a parallel with cosmology, in which the existence of an energetic background field is required to explain some cosmological phenomena, such as, for instance, the accelerated expansion of the universe by "dark energy". To understand this phenomenon within Einstein's framework for gravity, established by his theory of General Relativity, the dark energy is considered as being embodied by the Lambda parameter in his Field Equation. This Lambda parameter influences the space-time curving of the universe and modifies Newton's gravity law on top of the Schwarzschild solution for $\Lambda=0$. Although far from trivial, under particular constraints it appears possible to derive from Einstein's Field Equation a meaningful wave function for the bosonic central force, such that,

$$
-\frac{\partial^{2}}{c^{2} \partial t^{2}}(r \Phi)+\frac{\partial^{2}}{\partial r^{2}}(r \Phi)+\lambda^{2}(r \Phi)=-r(4 \pi G M) \delta^{3}(r) U(t),
$$

in which $\lambda^{2}=2 \Lambda$.

The constraints are twofold. The first one has to with the recognition that space is not empty, but, instead, behaves as a molecular fluidum in thermodynamic equilibrium. This allows removing an irrelevant bias, which, in fact, is an equivalent for renormalization as known within the context of the Standard Model of particle physics. As discussed in [2], this awareness explains why the well-known "Cosmology Constant catastrophe" [28] does not exist. The second element is the restriction to a spatial range of validity between a low spatial limit $r_{L}$ and a high spatial limit $r_{H} \lambda \approx 6$. The derivation of (37) can be found in [2]. Because of its vital importance in the context of this text, it is instructive to emphasize its difference from the common solutions of Einstein's Field Equations with inclusion of $\Lambda$ for 
pointlike sources in spherically symmetric space-time, provided by the Schwarzschild-de Sitter metric, also known as Kottler-metric $[39,40]$. Recognizing that the potential field $\Phi$ is a tiny modulation of the metric allows the removal of an irrelevant bias, thereby obtaining a valid wave equation for a weak field $\Phi$ showing a gradual move from $\Lambda=0$ to $\Lambda \neq 0$.

The sign of $\Lambda$ is highly relevant. Einstein's Equation leaves different options: positive, negative or zero. A positive sign would make the field spatially exponentially decreasing. A negative sign allows a spatially harmonic behavior under gradual $(1 / r)$ decrease. As shown in [2] in more detail: such a behavior results in a slight increase of the gravitational acceleration thereby modifying the Newtonian gravitation to the extent that it explains the excessive velocity of orbiting objects in galaxies, known as the dark matter effect. This dark matter effect is empirically expressed by Milgrom's acceleration constant $a_{0}$, which appears being a constant of about $a_{0} \approx 1.25 \times 10^{-10} \mathrm{~m} / \mathrm{s}^{2}$ in (probably) all galaxies. Application of (37) to orbital objects in galaxies reveals that Einstein's Lambda can be related with the Milgrom's acceleration constant $a_{0}$ as

$\lambda^{2}=2 \Lambda=\frac{2 a_{0}}{5 M G}$

in which $M$ is the mass of the galaxy.

Unlike what is commonly believed, Einstein's Lambda should not be seen as a constant of nature, but, instead, as a covariant integration constant, independent of space-time coordinates, which may assume different values that depend on the scope of the cosmological system under consideration. At the level of the universe assumes a value that may justify the common characterization as Cosmological Constant. In fact, Milgrom's acceleration constant $a_{0}$ is the better one, because the extension from the galaxy view to the cosmological view reveals that [2],

$a_{0}=\frac{15}{4} \Omega_{B} \frac{c^{2}}{c t_{H}}$

in which $\Omega_{B}(\approx 0.0486)$ is the relative amount of baryonic matter in the universe and in which $t_{H}$ is the Hubble time or life time of the universe ( $\approx 13.8$ Gyear). These results have been derived under adoption of Debije's theory of field shielding due to a background fluid of polarizable dipoles $[30,2]$. With the difference, though, of the polarization direction of the dipoles as a consequence of the different signs in $\Lambda$ between gravity and electromagnetism. The polarization density $P_{g 0}$, i.e. the number of polarizable dipoles per unit of volume in the cosmological background field, calculated similarly as will be shown below for the nuclear background field, appears being,

$$
P_{g 0}=\frac{a_{0}}{20 \pi G}
$$


Conceiving these dipoles as the darks that constitute the background field and furthermore supposing that these darks are Dirac particles with a polarizable dipole moment with the minimum possible magnitude $h / 2 c$, the volume density of darks is found as,

$N / \mathrm{m}^{3}=\frac{a_{0}}{20 \pi G}\left(\frac{2 c}{\hbar}\right) \approx 1.710^{14}$ particles per cubic nanometer.

So far, a summary of the cosmological background field. What about the nuclear background field? Recognizing the parallel and the correspondence between (35) and (13), there is no reason why space-time curving would be restricted to common massive energy. Accepting the RCMT magnetic monopole as the ultimate energetic source, it is fair to suppose that this source curves space-time similarly as a gravitational pointlike source does. There is a difference, though, in the sign of $\lambda^{2}$. As noted before, the theoretical derivation leaves the sign open to the physical interpretation for the origin of $\Lambda$, on which Einstein's theory does not give the answer. The physical interpretation just summarized has to do with the nature of the energetic constituents that assemble the energetic background fluid. Applying in both cases Debije's model of polarisable dipoles (almost mass less), the bosonic field is either enhanced or shielded. As argued, there is reason to suppose that in the gravity case the bosonic field is enhanced, because this would explain the dark matter effect in the gravitational objects orbiting in galaxies, while there is reason to suppose that in the nuclear case the bosonic field is shielded because of its limited range. In other words: whereas baryonic kernels are attracting on the background dipoles, the RCMT monopoles are repelling on the very same background dipoles. Let us see if we may find proof.

\section{Identifying the gravitational background field with the nuclear background field}

Applying (37), under condition of $\Lambda$ with negative sign, to the field of a Gilbertian magnetic monopole, we have in static condition in terms of Poisson's equation,

$$
\nabla^{2} \Phi-\lambda^{2} \Phi=-\mu_{0} \rho_{0}(r) ; \rho_{0}(r)=Q_{m} \delta^{3}(r)
$$

in which $Q_{m}$ is the magnetic charge of the monopole. Let us rewrite (42) as,

$$
\nabla^{2} \Phi=-\mu_{0} \rho(r) ; \rho(r)=Q_{m} \delta^{3}(r)-\rho_{D}(r) ; \rho_{D}(r)=\frac{\lambda^{2}}{\mu_{0}} \Phi(r)
$$

In Debije's theory of electric dipoles [29,30,31,32],

$$
\rho_{D}(r)=-\nabla \cdot \mathbf{P}_{\mathbf{g}}
$$

The vector $\mathbf{P}_{\mathbf{g}}$ is the dipole density. From (43) and (44),

$$
\rho_{D}=\frac{1}{r^{2}} \frac{\mathrm{d}}{\mathrm{d} r}\left\{r^{2} P_{g}(r)\right\}
$$


Assuming that in the static condition the space fluid is eventually fully polarized by the field of the pointlike source, $P_{g}(r)$ is a constant $P_{g 0}$. Hence, from (45),

$\rho_{D}(r)=2 \frac{P_{g 0}}{r}$

Taking into account that to first order,

$\Phi(r)=\frac{\mu_{0}}{4 \pi} \frac{Q_{m}}{r}$

we have under consideration of (43) and (46),

$\rho_{D}(r)=-\frac{\lambda^{2}}{\mu_{0}} \frac{\mu_{0}}{4 \pi} \frac{Q_{m}}{r}=\frac{2 P_{g 0}}{r} \rightarrow 2 P_{g 0}=-\frac{\lambda^{2}}{4 \pi} Q_{m}$

Supposing that the Higgs field is built up by elementary Gilbertian dipoles with dipole moments,

$p_{m}=q_{m} \hbar / 2 m_{d} c$

and that $N$ is the number of dipole moment carrying particles per unit of volume, we have from (47) and (48), ,

$\lambda^{2} \frac{Q_{m}}{4 \pi}=2 N p_{m}=2 N \frac{q_{m} \hbar}{2 m_{d} c} \rightarrow \frac{q_{m}}{Q_{m}}=\frac{\lambda^{2}}{4 \pi N} \frac{m_{d} c^{2}}{\hbar c}$.

The view that the energetic background field of particle physics coincides with the gravitational background field to the ultimate that the gravitational $\Lambda$ has the same magnitude as the particle physics $\Lambda$ has the implication that magnetic charges scale with $\lambda^{2}=2 \Lambda$, similarly as gravitational massive kernels do. Hence,

$\frac{q_{m}}{Q_{m}}=\frac{m_{d}}{m_{q u}}$

in which $m_{q u}$ is the bare mass of the quark. From (47) and (48),

$m_{q u} c^{2}=m_{q u}^{\prime}=4 \pi N \frac{\hbar c}{\lambda^{2}}$

From (12), we have $\lambda=m_{H}^{\prime} / 2 \hbar c$, in which $m_{H}^{\prime}$ is the energy of the Higgs boson. This relationship has been established in the center-of-mass frame of the archetype meson under neglect of the spin-spin interactions. Considering that the pion with its lab frame rest mass $m_{\pi} \approx 140 \mathrm{MeV} / \mathrm{c}^{2}$, decays by the weak interaction $m_{W} \approx 80.4 \mathrm{GeV} / \mathrm{c}^{2}$, we may consider the 
mass of the pion as the non-relativistic correction of the weak interaction boson. This allows to calculate the lab frame value of $\lambda$ as

$\lambda=\frac{m_{H}^{\prime}}{2 \hbar c}=\frac{m_{H}^{\prime}}{m_{W}^{\prime}} \frac{m_{W}^{\prime}}{2 \hbar c} \rightarrow \lambda=\frac{m_{H}^{\prime}}{m_{W}^{\prime}} \frac{m_{\pi}^{\prime}}{2 \hbar c}$.

Hence from (51) and (52)

$m_{q u}^{\prime}=4 \pi N \hbar c\left(\frac{2 \hbar c}{m_{\pi}^{\prime}} \frac{m_{W}^{\prime}}{m_{H}^{\prime}}\right)^{2}$

From (53) and (41) we have eventually for the quark's massive energy,

$m_{q u}^{\prime}=4 \pi \hbar c \frac{a_{0}}{20 \pi G} \frac{2 c}{\hbar}\left(\frac{2 \hbar c}{m_{\pi}^{\prime}} \frac{m_{W}^{\prime}}{m_{H}^{\prime}}\right)^{2}=\frac{8}{5} \frac{a_{0} c^{2}}{G}\left(\frac{\hbar c}{m_{\pi}^{\prime}} \frac{m_{W}^{\prime}}{m_{H}^{\prime}}\right)^{2} \approx 1.35 \mathrm{MeV}$

This bare mass value for the quark derived from equating the energetic background field of gravity with the energetic background field of quantum physics is somewhat lower than the bare mass value of the quark derived from the Maxwellian model discussed in paragraph 5 .

How to explain the difference? To answer this question, it has to be emphasized once more that the semantics of the mass values of quarks should be interpreted with care. Prior to the introduction of the lattice QCD theory, the quark masses have been assessed as constituent masses empirically calculated from the rest masses of the hadrons after full decay. As a result, the constituent masses of the quarks in mesons show different values from the constituent masses of the quarks in baryons. These masses are predominantly determined by interaction energies, thereby hiding the true energy erupting mass kernels. In the author's concept, the Maxwellian $3.9 \mathrm{MeV} / \mathrm{c}^{2}$ is part of the pion's rest mass and the 1.35 $\mathrm{MeV} / \mathrm{c}^{2}$ is the bare gravitational mass of the quark. While the Maxwellian $3.9 \mathrm{MeV} / \mathrm{c}^{2}$ "running mass" plays a role in the details of the mass spectrum of the hadrons, the gravitational $1.35 \mathrm{MeV} / \mathrm{c}^{2}$ "bare mass" is irrelevant for the mass spectrum.

\section{Discussion}

\section{Gravitational Constant}

Back in 2011, the author of this article has derived an expression for the Gravitational Constant $G$ in terms of quantum mechanical quantities [8]. The expression allows a successful numerical justification. The derivation was based upon a structural model of a quark and an antiquark, more or less similar to figure 2. As explained in this article once more, the structure represents an anharmonic quantum mechanical oscillator. In its center, one recognizes a vibrating amount of baryonic energy that originates from the non-baryonic energy of the composing quark kernels. The awareness that this baryonic amount of energy must be the right-hand term in Einstein's Field Equation, as built up from the potential field of the composing quarks, has led to the quoted expression. The 2011 quark model, though, was quite hypothetical. Not more than a pointlike source of nuclear energy of unknown kind, 
characterized by a static potential field, similar to eq. (3), with no other justification but a mathematical manipulation on the Lagrangian expression of the Higgs field. Whereas the $1 / x$ term in (3) can be readily interpreted as the contribution from a classical field, the $1 / x^{2}$ contribution as well as the exponential decay term $\exp (-\lambda x)$ were just adopted as features of the hypothetical Higgs field, without a clear physical interpretation. In a sequence of articles, although certainly not flawless, the virtue of the model has been demonstrated by successful calculations on the mass spectrum of mesons and baryons. Unfortunately, the model did not gain any credit, because of its hypothetical nature and because it was considered of being in conflict with the well proven Standard Model of particle physics. In spite of its numerical proof, the expression for the Gravitational Constant has been ignored, even so after a later more substantial publication in 2016 [10]. It sadly resulted into a place on Jean de Climont's list of dissident scientists [37].

\section{The liquid drop model}

The rather convincing relationship between gravity and quantum physics as shown in the verifiable Gravitational Constant expression challenged me trying to give a better motivation for the quark's model but just the hypothetical one derived from the Higgs field Lagrangian. This required finding an explanation for the field component $1 / x^{2}$ and for the field decay term. This exponential decay must be due to the field shielding of a monopole potential due to some kind of background energy. The field component $1 / x^{2}$ evokes the suggestion that it must be due to the presence of a dipole. More challenging than finding an explanation for the exponential decay term in the quark's potential field, is finding an explanation for the origin of a polarisable dipole contribution in a scalar field. Considering that a quark is a Dirac particle, such a dipole would be rather curious, because the archetype Dirac particle, like an electron, has a magnetic dipole, which is not polarisable in a scalar potential field. Dirac's theory, though, shows two dipole moments. Next to a first one, real valued to the amount of $\left(e \hbar / 2 m_{0}\right)$, known as the (anomalous) magnetic dipole moment, it shows a second one, an electric dipole moment, imaginary valued to an amount of $\left(i e \hbar / 2 m_{0} c\right)$. The latter is one of the two anomalies of Dirac's theory, pointed out by Dirac himself. He noticed a negative energy solution next to a positive energy solution. And he noticed a real magnetic moment next to an imaginary electrical dipole moment. About the first item he remarked that the problem would disappear if the electron would change its polarity, but that "this is a phenomenon not yet observed". About the second item he remarked that he doubted about the physical meaning of an imaginary electrical dipole moment. Whereas he welcomed the real magnetic dipole moment as a confirmation of a known physical phenomenon ("the spinning electron"), he suggested that the imaginary electrical dipole moment would be a mathematical artefact as a result of "the artificial multiplication of his wave function to create an Hamiltonian that resembles the one of previous theories". In fact, however, the negative energy solution is a result of this artificial multiplication as well. It gives reason for considering a potential generalization of Dirac's approach, like discussed in paragraph 3 of this article.

The generalization has shown the potential viability that a quark can be conceived as a Dirac particle of a particular kind, denoted as third. The most essential property of this particle is the nuclear equivalent of the electric dipole moment. Whereas the electric dipole moment of an electron type Dirac particle is imaginary, it is real for the "third". As a consequence, the 
quark is polarisable in a scalar potential field. This property gives an adequate explanation for the "gluing" of quarks in hadrons. Like discussed in paragraph 3, the justification for the potential existence of "thirds" is based upon the recognition that Dirac's heuristic transformation rule on rest mass, spatial momenta and temporal momentum into operators on wave function components allows a generalization into three basic types. Whereas a second type next to the electron type, known as tachyon, has been accepted as theoretically feasible (although not yet proven by physical evidence), the "third" with its two real dipole moments has not been proposed by other authors.

\section{Einstein's $\Lambda$ and the Higgs field}

The exponential decay of the quark's potential field must be due to some kind of background energy. And this background energy must be due to elementary constituents. Apparently, these constituents shield the quark's field similarly as the field of an electric charge in an ionic plasma is shielded as the consequence of the Debije effect. This occurs if the elementary constituents are polarisable dipoles. This evokes the suggestion that the constituents are elementary Dirac particles of the third kind. Although quarks and such constituents both are Dirac particles of the third kind, sharing the property of possessing of a polarisable dipole moment in a scalar potential, they must be quite different in size and in bare mass. Curiously, an energetic background field is omni-present in cosmology as well. It must be present to explain the accelerated expansion of the universe. It is embodied by the Lambda in Einstein's Field Equation. This awareness has resulted in my attempt to linearise Einstein's Field Equation with inclusion of $\Lambda$. As discussed in paragraph 6 , this linearization slightly modifies the Newtonian gravitation law by a damped very weak oscillatory term. However, whereas in particle physics the background field is of a suppression nature that limits the effective range of nuclear forces, in cosmology the background field is enhancing, although very slightly, the Newtonian gravity strength such as becomes apparent in the dark matter effect. It gives a physical explanation to the mathematical $\Lambda$ term in Einstein's equation by proving that it shows up as the result of vacuum polarization due to polarisable dipole moments of elementary energetic constituents, dubbed as darks. It is not a big step to suppose that these darks are the same as the energetic constituents of the nuclear background field. This implies that the background field of particle physics, hypothetically defined in the Standard Model as the Higgs field, is the same as the cosmological background field embodied by $\Lambda$ in the $\Lambda$ CDM standard model of cosmology. The acceptance of this view implies that darks can be polarized by a scalar gravitational field as well as by a scalar nuclear field. While in gravity the polarization is field enhancing, it is field shielding in the nuclear case. Unlike in gravity and in particle physics, the darks don't feel a polarization influence from scalar electric fields. The vacuum is fully transparent for classical electromagnetism. The dark matter theory discussed in paragraph 6 enables to calculate the particle density of darks. Relating this density with the known critical mass density of the universe reveals that the darks are virtually mass less particles with a calculated energy of about $\approx 310^{-32} \mathrm{eV}$ [2]. Like discussed in the paragraph 7, the assumption that the cosmological darks are the same as the constituents of the Higgs field allows to use their particle density for calculating the bare mass (not to be confused with the constituent mass) of the basic $u / d$ quark as $1.35 \mathrm{MeV} / \mathrm{c}^{2}$.

This explanation of the dark matter phenomenon as a consequence from the dark energy meets criticism because of the denial that Einstein's $\Lambda$ is a constant of nature, usually 
identified as the Cosmological Constant. Instead, my analysis has been based upon the view that $\Lambda$ is a covariant integration constant that may have different values depending on the scope of a cosmological system under consideration. Only at the level of the universe it is justified to identify $\Lambda$ as the Cosmological Constant indeed. This awareness is based upon Einstein's note in his 1916 article that he equated an integration constant as zero (see footnote on p.804 in ref. [38]). Anyhow, the result of this is an explanation for the far field behavior of classical fields of energy, in which the polarity sign of a non zero $\Lambda$ is responsible either for a exponential decay or for an initial enhancement of the field. Similarly, I may expect that the concept of a third mode of Dirac's particle, next to the bradyon (= electron type) and tachyon will be subject to serious criticism as well and that a more fundamental analysis but the one put forward in this article, would be required. Let me summarize some of my reasons for its existence instead.

\section{Unsolved problems solved}

1. The quark-antiquark model shown in figure 2 has allowed to express the Gravitational Constant $G$ into quantum mechanical quantities with a successful numerical proof $[8,10]$.

2. Different from a theoretical axiomatic concept, isospin is a physical attribute associated with the quark's polarisable dipole moment [5].

3. The number of elementary quarks can be reduced to a single basic archetype [5]

4. The big gap between the rest masses of the $(u / d, s, c, b)$ quarks on the one hand hand and the topquark on the other hand is a consequence of the loss of binding energy between the quarks [5].

5. The massive rest mass energies of the Higgs boson, the $W / Z$ bosons and the topquark can be assessed by theory. Experimental evidence is a confirmation instead of empirical axioms [5].

6. The gluon-quark relationship is the equivalent of the photon-electron relationship [5].

7. The mathematical axiomatic SU(2) and SU(3) gauges of particle physics theory can be replaced by physically based gauges similar to the electromagnetic $U(1)$ gauge [5].

8. Explains the non-existence of the "Cosmological Constant catastrophe".

All this relies upon the particular characteristics of a quark, modeled as a Dirac particle in its "third" mode. This mode is associated with a particular free state of energy $W$, different from the Einsteinean energy, but subject to the dispersion relationship $W^{2}=\left(m_{0} c^{2}\right)^{2}-|\mathbf{p}|^{2} c^{2}$. This is different from the one shown by an electron and a pointlike mass moving in empty space described in classical electromagnetism and in Newtonian gravity. in the concept summarized in this article, space is filled with a fluidum, consisting of tiny polarisable dipoles. As long as this fluidum is not polarized, space is in a state of maximum symmetry and entropy. It then just behaves fully transparently for an energetic source, such as an electric monopole does. As we have discussed, unlike an electric monopole, a nuclear monopole, like a quark, is able to polarize the fluid. This polarization in conjunction with the quark's dispersion relationship is the equivalent of the spontaneous symmetry breaking of the quark's physical system as axiomatically defined in the Standard Model of particle physics. 


\section{Origin of electric charge}

The list just shown is just a small one from many more. In this theoretical concept, the quark is a classical pointlike monopole spreading a potential field, just akin to the one from a pointlike electric charge in electromagnetism and to the one from a baryonic massive kernel in Newtonian gravity. The physical nature of the quark monopole is a generic kind of nuclear energy expressed in terms of a potential $\Phi$ with the dimension of energy. As discussed in paragraph 4, rather than defining the monopole strength in terms of energy, one might equivalently define its strength in terms of a nuclear charge, similarly as done in electromagnetism. This consideration evokes the challenge to search for a possible relationship between electric charge and nuclear charge. Whereas the origin of baryon charge (= gravitational mass) is clearly the result from the conversion of nuclear energy (nuclear charge) in the (an)harmonic oscillator structure composed by the quark-antiquark bond, the origin of electric charge different from just axiomatic, is not clear. As discussed in paragraph 5, the identification of nuclear charge as the charge of Comay's monopole, reveals the origin of electric charge. It can be traced back to the different magnetic monopole (quark) characteristics as compared with those of an electric one (electron). Whereas the magnetic monopole has two real dipole moments, the electron monopole has a single real one, because the second one is imaginary. The origin of electric charge can now be explained as the source of the magnetic dipole moment of the quark. The analysis in paragraph has shown that such fits numerically well. The model denies a asymmetrical split of the elementary charge. This is in agreement with the view that electric charge should be considered as an holistic attribute of hadrons as a whole, instead of composed by fragmented contributions [5]. Up to now Comay's monopole model for quarks has not get any credit. Instead, it resulted into Comay's place on Jean de Climont's list of dissident scientists [36].

It may seem as if the theory as summarized in this article is in conflict with the Standard Model of particle physics. This imposes a problem indeed, because the Standard Model is regarded as being well proven by an overwhelming amount of experimental evidence of its correctness. In fact, there might be no conflict if one wishes to accept that there is a need to gain understanding a physical basis for the underlying axioms that are accepted in present theory. It has to do with the basic question "what glues the quarks together?" in relation with the object to construct a covariant theory based on well-defined gauges. This objective has led to the $\mathrm{U}(1) \otimes \mathrm{SU}(2) \otimes \mathrm{SU}(3)$ Standard Model. However, whereas the $\mathrm{U}(1)$ gauging as inherited from Dirac's electron theory and Einstein's General Relativistic theory is based upon a well-understood physical mechanism, the $\mathrm{SU}(2)$ and $\mathrm{SU}(3)$ gauging is purely axiomatic (in fact heuristic), because of the lack of knowledge of a physical mechanism that glues two or three elementary particles together. There is nothing wrong of course with an axiomatic basis. In fact, the more axioms, the more accurate a theory is. But it is true as well that mathematically conceived axioms may hide physical interrelationships. It has been shown in this article that the force that glues two and three elementary particles together is due to a unique property of a quark, namely its polarisable dipole moment in a scalar field next to its angular dipole moment. Whereas the latter is known as nuclear spin, the former should be recognized as isospin. This gives a physical justification for the $S U(2)$ and $S U(3)$ axioms including weak isospin, electroweak unification, gluons and the like. That the quark possesses a polarisable dipole moment in a scalar field is not trivial, because it is commonly accepted that the quark is a Dirac particle. Whereas Dirac's theory of electrons reveals a 
second dipole moment next to the angular dipole moment or spin indeed, this dipole moment shows up as an imaginary quantity and is therefore physically non-existing. However, as argued in this article, the quark is a Dirac particle of a non-electron type. More particularly, it is a pseudo-tachyon (xenon ? = "stranger"), described by a set of gammamatrices different from the canonical ones.

Anyhow, joining the theories of two dissident scientists, summarized in this discussion, may give a clue to a theory that unifies quantum mechanics, electromagnetism and gravity, such as summarized in the following corollary.

\section{Corollary}

The four fundamental physical forces can be unified in a single theory. Essential elements of the theory are:

1. The quark is a pseudo-tachyon. i.e., an unrecognized Dirac particle that has, next to the well-known real dipole moment associated with the elementary angular momentum $\boldsymbol{h}$, a second real dipole moment associated with an elementary linear dipole moment $\boldsymbol{h} / \boldsymbol{c}$, which, unlike as in the case of electrons, is polarisable in a scalar potential field. Its theory can be found in $[1,10]$

2. The quark is an RCMT monopole. This monopole is different from the well-known Dirac monopole, because its Maxwellian definition is disjunct from Dirac's one. Its theory can be found in [9].

3. Because the quark is a pseudo-tachyon as well, it has a real magnetic dipole moment. This magnetic dipole moment defines an elementary amount of electric charge associated with the quark, such as described in this article.

4. The quark's RCMT magnetic charge spreads a Coulomb-type nuclear field to which other quarks may couple with a coupling factor equal to their own magnetic charge.

5. The quark's RCMT field is shielded by a background field, known as the Higgs field. It can be conceived as an energetic plasma of tiny magnetic dipoles in a similar way as an electric charge is shielded by the polarization of electric dipoles in an ionic plasma (Debije effect). The quark's electric field from its elementary charge is unaffected by the polarization of the energetic background field.

6. Bonds of three quarks (baryons) and quark-antiquark (bonds) behave as quantummechanical oscillators that convert the RCMT monopole fields of the pseudo-tachyon quarks into baryonic energy. The vibration energy of the center-of-energy is the baryonic manifestation of the non-baryonic energy of the quarks $[5,10]$

\section{Conclusion}


Whereas the theory developed in [5] has shown the unification between weak interaction, strong interaction and gravity from the concept of a single archetype quark, the unification is completed with electromagnetism by the hypothesis that the archetype quark is an RCMT monopole [9]. The quark is the energetic source of all four fundamental physical forces.

\section{Appendix: The second dipole moment of the quark}

The simple model as described in the introduction relies on the presupposed quark's property of showing nuclear equivalents for the magnetic dipole moment and the electric dipole moment of electron-type Dirac particles. Whereas the viability of an equivalent for the magnetic dipole moment, related with the elementary angular momentum $\hbar$ can be readily understood, it is not the case for an equivalent of the electric dipole moment, related with the position momentum $\hbar / c$. While for common Dirac particles the magnetic dipole moment is a real value, the electric dipole moment is an imaginary value, hence non-existing [12]. This implies that if the quark shows two real dipole moments like described in the introduction above, it can't be a common Dirac particle. If it is a Dirac particle indeed, it must be an uncommon one. Let us try finding if such an uncommon type with the desired property would be feasible from a theoretical point of view.

To do so, let us start from the canonic format of Dirac's equation as captured by,

$\left(\mathrm{i} \hbar \gamma^{\mu} \partial_{\mu} \psi-m_{0} c \psi\right)=0 \rightarrow\left(\hbar \gamma^{\mu} \frac{\partial_{\mu} \psi}{\mathrm{i}}-\frac{1}{\mathrm{i}^{2}} m_{0} c \psi\right)=0$

It can be rewritten after division by $m_{0} c$, in terms of wave function operators as,

$\left[\gamma_{0} \hat{p}_{0}^{\prime}+\left(\bar{\gamma} \cdot \hat{\mathbf{p}}^{\prime}\right)+I_{4}\right] \psi=0$

in which $\hat{\mathbf{p}}^{\prime}=\hat{\mathbf{p}}^{\prime}\left(\hat{p}_{1}, \hat{p}_{2}, \hat{p}_{3}\right)$ with

$\hat{p}_{i}^{\prime}=\frac{1}{m_{0} c} \frac{\hbar}{\mathrm{i}} \frac{\partial}{\partial x_{i}}$ and $\hat{p}_{0}^{\prime}=\frac{1}{m_{0} c} \frac{\hbar}{\mathrm{i}} \frac{\partial}{\partial c \tau}$

and in which $I_{4}$ is the $4 \times 4$ identity matrix.

Note that the variables are signed by 'to emphasize their normalization on $m_{0} c$. Note also that the temporal parameter is written as proper time $\tau$ to emphasize the (special) relativistic nature of Dirac's equation in free space. Rewriting (A-2) in the Weyl format gives,

$$
\left[\begin{array}{cc}
I & 0 \\
0 & -I
\end{array}\right]\left[\begin{array}{c}
\hat{p}_{0}^{\prime} \psi \\
\hat{p}_{0}^{\prime} \chi
\end{array}\right]+\left[\begin{array}{cc}
0 & \bar{\sigma} \\
-\bar{\sigma} & 0
\end{array}\right]\left[\begin{array}{c}
\hat{\mathbf{p}}^{\prime} \psi \\
\hat{\mathbf{p}}^{\prime} \chi
\end{array}\right]+\left[\begin{array}{cc}
I & 0 \\
0 & I
\end{array}\right]\left[\begin{array}{l}
\psi \\
\chi
\end{array}\right]=0,
$$

in which $\bar{\sigma}=\bar{\sigma}\left(\sigma_{1}, \sigma_{2}, \sigma_{3}\right)$ is the Pauli vector with the three Pauli matrices. 
As known, Dirac's equation is based upon a heuristic elaboration of the Einsteinean energy expression under use of particular properties of the $\gamma$ matrices. These properties can be summarized as,

$\gamma_{\mu} \gamma_{\nu}+\gamma_{\nu} \gamma_{\mu}=0$ if $\mu \neq v$; and $\gamma_{0}^{2}=1 ; \gamma_{i}^{2}=-1 ; \beta^{2}=1$

in which $\beta$ is the last matrix term in (A-2). Recognizing that the last term in the left hand part of (A-2) represent a matrix $\beta$ and that $(A-1)$ is valid for a plus sign in front of $m_{0}$ as well, one should add in fact,

$\gamma_{\mu} \beta \mp \beta \gamma_{\mu}=0 ; \beta= \pm 1$

which is trivial as long as $\beta$ is an Identity matrix. The very same properties are met if $(A-4)$ is modified into,

$$
\left[\begin{array}{cc}
I & 0 \\
0 & -I
\end{array}\right]\left[\begin{array}{c}
\hat{p}_{0}^{\prime} \psi \\
\hat{p}_{0}^{\prime} \chi
\end{array}\right]+\left[\begin{array}{cc}
0 & \bar{\sigma} \\
-\bar{\sigma} & 0
\end{array}\right]\left[\begin{array}{c}
\hat{\mathbf{p}}^{\prime} \psi \\
\hat{\mathbf{p}}^{\prime} \chi
\end{array}\right]+\mathrm{i}\left[\begin{array}{cc}
0 & I \\
I & 0
\end{array}\right]\left[\begin{array}{l}
\psi \\
\chi
\end{array}\right]=0
$$

Note that the $\beta$ is modified from the $4 \times 4$ identity matrix into the imaginary value of the "fifth" gamma matrix $\gamma_{5}$. The two representations (A-6) and (A-4) are equivalent. Both represent the common electron-type Dirac particle with a real magnetic dipole moment and an imaginary electric dipole moment. If $\beta$ would have been modified into the real value of $\gamma_{5}$, we would have obtained the tachyon format, which reads as,

$$
\left[\begin{array}{cc}
I & 0 \\
0 & -I
\end{array}\right]\left[\begin{array}{c}
\hat{p}_{0}^{\prime} \psi \\
\hat{p}_{0}^{\prime} \chi
\end{array}\right]+\left[\begin{array}{cc}
0 & \bar{\sigma} \\
-\bar{\sigma} & 0
\end{array}\right]\left[\begin{array}{c}
\hat{\mathbf{p}}^{\prime} \psi \\
\hat{\mathbf{p}}^{\prime} \chi
\end{array}\right]+\left[\begin{array}{cc}
0 & I \\
I & 0
\end{array}\right]\left[\begin{array}{l}
\psi \\
\chi
\end{array}\right]=0 .
$$

This tachyon format is studied in the context of the hypothetical existence of superluminal particles [13]. It does meet the constraint (A-5a), but it violates constraint (A-5b). Instead it meets,

$$
\gamma_{\mu} \beta+\beta \gamma_{\mu}=0 ; \beta^{2}=-1
$$

Note the subtle difference between $(A-5 b)$ and $(A-8)$. The dipole moments of the tachyon are similar to those of the electron-type: the equivalent magnetic one is real and the equivalent electric one is imaginary.

Both dipole moments are real for a third modification of Dirac's particle $[3,4]$. This modification reads as,

$\mathrm{i}\left[\begin{array}{cc}I & 0 \\ 0 & -I\end{array}\right]\left[\begin{array}{c}\hat{p}_{0}^{\prime} \psi \\ \hat{p}_{0}^{\prime} \chi\end{array}\right]+\left[\begin{array}{cc}0 & \bar{\sigma} \\ -\bar{\sigma} & 0\end{array}\right]\left[\begin{array}{c}\hat{\mathbf{p}}^{\prime} \psi \\ \hat{\mathbf{p}}^{\prime} \chi\end{array}\right]+\left[\begin{array}{cc}0 & I \\ I & 0\end{array}\right]\left[\begin{array}{l}\psi \\ \chi\end{array}\right]=0$. 
As compared with the electron-type (A-4), the $\gamma_{0}$ matrix is made imaginary. It meets the constraints,

$$
\gamma_{\mu} \gamma_{v}+\gamma_{v} \gamma_{\mu}=0 \text { if } \mu \neq v ; \gamma_{\mu} \beta+\beta \gamma_{\mu}=0 ; \gamma_{0}^{2}=-1 ; \gamma_{i}^{2}=-1 ; \beta^{2}=-1
$$

To understand the violations of the constraints $(A-5)$ and the modifications into $(A-8)$ and $(A-$ $10)$, it is instructive to solve the various formats $(A-6),(A-7)$ and $(A-9)$ of Dirac's equation. In full expansion mode, $(\mathrm{A}-9)$ reads as

$$
\mathrm{i}\left[\begin{array}{cccc}
1 & 0 & 0 & 0 \\
0 & 1 & 0 & 0 \\
0 & 0 & -1 & 0 \\
0 & 0 & 0 & -1
\end{array}\right]\left[\begin{array}{c}
\hat{p}_{0}^{\prime} \psi_{0} \\
\hat{p}_{0}^{\prime} \psi_{1} \\
\hat{p}_{0}^{\prime} \psi_{2} \\
\hat{p}_{0}^{\prime} \psi_{3}
\end{array}\right]+\left[\begin{array}{cccc}
0 & 0 & 0 & 1 \\
0 & 0 & 1 & 0 \\
0 & -1 & 0 & 0 \\
-1 & 0 & 0 & 0
\end{array}\right]\left[\begin{array}{c}
\hat{p}_{1}^{\prime} \psi_{0} \\
\hat{p}_{1}^{\prime} \psi_{1} \\
\hat{p}_{1}^{\prime} \psi_{2} \\
\hat{p}_{1}^{\prime} \psi_{3}
\end{array}\right]+\left[\begin{array}{cccc}
0 & 0 & 0 & -\mathrm{i} \\
0 & 0 & \mathrm{i} & 0 \\
0 & \mathrm{i} & 0 & 0 \\
-\mathrm{i} & 0 & 0 & 0
\end{array}\right]\left[\begin{array}{c}
\hat{p}_{2}^{\prime} \psi_{0} \\
\hat{p}_{2}^{\prime} \psi_{1} \\
\hat{p}_{2}^{\prime} \psi_{2} \\
\hat{p}_{2}^{\prime} \psi_{3}
\end{array}\right]+\left[\begin{array}{cccc}
0 & 0 & 1 & 0 \\
0 & 0 & 0 & -1 \\
-1 & 0 & 0 & 0 \\
0 & 1 & 0 & 0
\end{array}\right]\left[\begin{array}{c}
\hat{p}_{3}^{\prime} \psi_{0} \\
\hat{p}_{3}^{\prime} \psi_{1} \\
\hat{p}_{3}^{\prime} \psi_{2} \\
\hat{p}_{3}^{\prime} \psi_{3}
\end{array}\right]+\left[\begin{array}{cccc}
0 & 0 & 1 & 0 \\
0 & 0 & 0 & 1 \\
1 & 0 & 0 & 0 \\
0 & 1 & 0 & 0
\end{array}\right]\left[\begin{array}{c}
\psi_{0} \\
\psi_{1} \\
\psi_{2} \\
\psi_{3}
\end{array}\right]=0
$$

and written differently,

$$
\left[\begin{array}{cccc}
\mathrm{i} \hat{p}_{0}^{\prime} & 0 & \hat{p}_{3}^{\prime}+1 & \left(\hat{p}_{1}^{\prime}-\mathrm{i} \hat{p}_{2}^{\prime}\right) \\
0 & \mathrm{i} \hat{p}_{0}^{\prime} & \left(\hat{p}_{1}^{\prime}+\mathrm{i} \hat{p}_{2}^{\prime}\right) & -\hat{p}^{\prime}+1_{3} \\
-\hat{p}_{3}^{\prime}+1 & \left(\hat{p}_{1}^{\prime}-\mathrm{i} \hat{p}_{2}^{\prime}\right) & -\mathrm{i} \hat{p}_{0}^{\prime} & 0 \\
-\left(\hat{p}_{1}^{\prime}+\mathrm{i} \hat{p}_{2}^{\prime}\right) & \hat{p}^{\prime}+1_{3} & 0 & -\mathrm{i} \hat{p}_{0}^{\prime}
\end{array}\right]\left[\begin{array}{c}
\psi_{0} \\
\psi_{1} \\
\psi_{2} \\
\psi_{3}
\end{array}\right]=0
$$

Let $\psi=u_{\mu} \exp \{\mathbf{i}(\mathbf{k} \cdot \mathbf{r}-\omega t)\} ; \mathbf{k}=\mathbf{p} / \hbar ; \omega=W / \hbar$.

Applying (A-12) on (A-11) gives after some elaboration,

$$
\left[\begin{array}{cccc}
-\mathrm{i} W & 0 & c p_{3}+m_{0} c^{2} & c\left(p_{1}-\mathrm{i} p_{2}\right) \\
0 & -\mathrm{i} W & c\left(p_{1}+\mathrm{i} p_{2}\right) & -c p_{3}+m_{0} c^{2} \\
-c p_{3}+m_{0} c^{2} & -c\left(p_{1}-\mathrm{i} p_{2}\right) & \mathrm{i} W & 0 \\
-c\left(p_{1}+\mathrm{i} p_{2}\right) & c p_{3}+m_{0} c^{2} & 0 & \mathrm{i} W
\end{array}\right]\left[\begin{array}{l}
u_{0} \\
u_{1} \\
u_{2} \\
u_{3}
\end{array}\right]=0
$$

This homogeneous set of equations has the solution (A-12) indeed under the constraint of the determinant value

$$
W^{2}=\left(m_{0} c^{2}\right)^{2}-c^{2}|\mathbf{p}|^{2}
$$

The canonical equations (A-2) or (A-4) show the same solution (A-12), but different from (A14), under the constraint,

$$
W^{2}=E_{W}^{2}=c^{2}|\mathbf{p}|^{2}+\left(m_{0} c^{2}\right)^{2}
$$

The tachyon equation (A-7) shows solution (A-12) for

$$
W^{2}=c^{2}|\mathbf{p}|^{2}-\left(m_{0} c^{2}\right)^{2}
$$


For a meaningful wave function, $\omega$ and $\mathbf{k}$, hence $W$ and $\mathbf{p}$, must be real. Hence, let us consider the condition (A-14) more closely. It can be rewritten as,

$$
\frac{W^{2}}{\left(m_{0} c^{2}\right)^{2}}=1+\frac{(v / c)^{2}}{(v / c)^{2}-1}=0 \rightarrow \frac{W^{2}}{\left(m_{0} c^{2}\right)^{2}}=\frac{1-2(v / c)^{2}}{1-(v / c)^{2}}
$$

The condition for the momentum p evolves as,

$$
\frac{c^{2}|\mathbf{p}|^{2}}{\left(m_{0} c^{2}\right)^{2}}=1-\frac{W^{2}}{\left(m_{0} c^{2}\right)^{2}}=1-\frac{1-2(v / c)^{2}}{1-(v / c)^{2}} \rightarrow \frac{|\mathbf{p}|}{m_{0} c}= \pm \sqrt{\frac{(v / c)^{2}}{1-(v / c)^{2}}}
$$

Hence,

$$
W= \pm m_{0} c^{2} \sqrt{\frac{1-2(v / c)^{2}}{1-(v / c)^{2}}} ;|\mathbf{p}|= \pm \frac{m_{0} v}{\sqrt{1-(v / c)^{2}}} .
$$

The similar elaboration for the tachyon format results into,

$$
W= \pm \frac{m_{0} c^{2}}{\sqrt{(v / c)^{2}-1}} ; \frac{|\mathbf{p}|}{m_{0} c}= \pm \frac{m_{0} v}{\sqrt{(v / c)^{2}-1}}
$$

The tachyon format shows real values for $W$ and $\mathbf{p}$ under superluminal conditions. It is a reason for speculations on the potential existence of superluminal particles. It is not meaningful under subluminal conditions, because the real values turn into imaginary ones. The properties of the "third" format, though, as shown by $(A-19,20)$ are real under subluminal conditions. The real value of its second dipole moment makes it of interest.

The interpretation of its $W$ as energy, shown by (A-14) implies that this energy decreases if it goes from rest into motion. This is contra-intuitive, because In true empty space one would expect the opposite, such as expressed by the Einsteinean energy expression (A-15) that holds for the canonical case. There is, however, no compelling reason why, in spite of its dimensional appearance, $W$ should be identical with the Einsteinean energy. The only thing that matters is its real value under subluminal condition, such as shown by (A-19). We are used to the Einsteinean energy relationship that says that a particle gains energy when it moves from rest into motion. This happens, however, only if additional energy is fed into the particle. In a conservative system, like an orbiting electron, this additional energy is given as initial state. The quark described by (A-9), though, shows the opposite: it seems to lose energy if it moves from rest into motion. It inherits its motional energy from its rest mass. This is, possibly, in its fundament, not essentially different from adding some initial energy to the rest mass like in the case of an orbiting electron. Like proven in $[3,4]$, such a particular Dirac particle has a polarisable dipole moment in a scalar field. The existence of such a dipole moment validates the structural quark model shown in figure 2 . 


\section{References}

[1] T. Padmanabhan, "The atoms of spacetime and the cosmological constant", J. Phys. Conf. Ser. 880012008 (2017)

[2] E. Roza, "The vacuum energy in the universe at the galaxy level, the cosmological level and the quantum level", www.preprints.org doi:10.20944/preprints202108.0159.v1 (2021)

[3] E. Roza, "On the second dipole moment of Dirac's particle", Found. of Phys. 50, 828 (2020)

[4] E. Roza, "On the second dipole moment of Dirac's particle", updated, www.preprints.org doi:10.20944/preprints202002.0275.v3

[5] E. Roza, "On the quark scaling theorem and the polarisable dipole moment of the quark in a scalar field", www.preprints.org, doi:10.20944/preprints202006.0304.v3

[6] C. Moskowitz, "Proton spin mystery gains a new clue", Scientific American, July 21, 2014

[7] G. Gamov, "Zur Quantentheorie des Atomkernes", Z. Physik 51, 204 (1928)

[8] E. Roza, E, "A hypothetical H-particle", Phys. Essays, 24, 1, 72 (2011).

[9] E. Comay, "The Regular Charge-Monopole Theory and Strong Interactions", Electr. J. of Theor. Phys., No.26, 93 (2012)

[10] E. Roza, "The gravitational constant as a quantum mechanical expression", Results in Phys., 6, 149 (2016)

[11] Griffiths, D.: Introduction to Elementary Particles, ISBN 3527406018, Wiley (2008)

[12] Dirac, P.A.M: "The quantum theory of the electron", Proc.R. Soc. Lond. A 117 (778), 610 (1928)

[13] U.D. Jentschura, "Dirac Hamiltonian with imaginary mass and induced helicicity", Journ. Modern Phys, vol. 3, issue 9, 887 (2012)

[14] G. Feinberg, "Possibility of Faster-Than-Light Particles", Phys. Rev., 159 , 1089 (1967)

[15] P. Dirac, "Quantised Singularities in the Electromagnetic Field", Proc. Royal Soc. A 133, 60 (1931)

[16] E. Comay, "Inherent Differences between Bound and Radiation Fields", Open Access Library J. 5,e4517 (2018)

[17] K.T. McDonald, "Comay's paradox: Do Magnetic Charges Conserve Energy", https://www.hep.princeton.edu/ modonald/examples/comay.pdf (2015)

[18] Ö. Zor, "On interactions of static magnetic fields", Journ. Electr. Eng., vol.70, no3, 253 (2019)

[19] E. Comay, "Spin, isospin and strong interaction dynamics", Progr. In Phys., 4, 55 (2011). [20] G.'t Hooft, " Agnetic monopoles in unified gauge theories", Nucl. Phys. B 79, 276 (1974)

[21] A. Polyakov, "Particle spectrum in quantum field theory".,JETP Lett. 20, 194 (1974)

[22] C. Dokos, T. Tomaras, 1980." Monopoles and dyons in the SU(5) model", Phys. Rev.

D 21, 2940 (1980)

[23] Y.M. Cho, "Physical implications of electroweak monopole", Philos Trans A Math Phys Eng Sci, 377, 2161, doi: 10.1098/rsta.2019.0038 (2019)

[24] J. Schwinger, "A Magnetic Model of Matter", Science 165, 3895 (1969)

[25] E. Comay, "Spin, isospin and strong interaction dynamics", Progr. In Phys., 4, 55 (2011)

[26] E. Roza, "The H-type quark and the baryons", Phys. Essays, 27, 3, 390 (2014)

[27] J. Ashman, EMC Collaboration (1988). "A measurement of the spin asymmetry and determination of the structure function $\mathrm{g} 1$ in deep inelastic muon-proton scattering". Physics Letters B. 206 (2) 
[28] R.J. Adler, B. Casey, O.C. Jacob, "Vacuum Catastrophe: An Elementary Exposition of the Cosmological Constant", Am. Journ. of Phys. 63 (7), 620 (1995)

[29] D. Hajdukovic, Astrophysics and Space Science, 334, vol.2, 215 (2011)

[30] P. Debye and E. Huckel, Physik. Zeitschrift, vol. 24, 9, 185 (1923)

[31] C.A. Gonano, R.E. Zich, M. Mussetta, Progr. in Electromagn. Res. B, 64, 83 (2015)

[32] E. Roza, "The vacuum paradox and its solution", Astrophys. and Space Sci., 364:73, doi.org/10.1007/s10509-019-3561-9 (2019)

]33] J.A. Frieman, M.S. Turner, D. Huterer, "Dark energy and the accelerating universe", Ann. Rev. Astronomy and Astrophys. 46, 385 (2008)

[34] P.J.E. Peebles, B. Ratra, Bharat (2003), "The cosmological constant and dark energy", Reviews of Modern Physics. 75 (2): 559, (2003)

[35] J. Schwichtenberg, Demystifying Symmetry Breaking, http//:jacobschwichtenberg.com, Aug.20, 2020

[36] M. Tanabashi et al. (Particle Data Group), "Review of Particle Physics", Phys. Rev. D 98 (3), 1 (2018)

[367 J. de Climont, "The worldwide list of dissident scientists",

https://www.academia.edu/37679452

[38] A. Einstein, Relativity: The Special and General Theory, H. Holt and Company, New York (1916, translation 1920)

[39] F. Kottler, Ann. Physik 56,361,401 (1918)

[40] Li-Feng Sun et al., Modern Phys. Lett. A 28, 1350114 (2013)

[41] E. Roza, "The spin-spin interaction mechanism between quarks and its impact on the mass spectrum of mesons", www.preprints.org doi:10.20944/preprints201701.0076.v5

(2022) 